\title{
Accuracy of ventilator-associated events for the diagnosis of ventilator-associated lower respiratory tract infections
}

\author{
Olivier Pouly ${ }^{1,2}$, Sylvain Lecailtel ${ }^{3}$, Sophie Six ${ }^{1}$, Sébastien Préau ${ }^{1}$, Frédéric Wallet ${ }^{4}$, Saad Nseir ${ }^{1,2^{*}}$ (D) \\ and Anahita Rouzé ${ }^{1}$
}

\begin{abstract}
Background: The aim of this study was to investigate the concordance between ventilator-associated events (VAE) and ventilator-associated lower respiratory tract infections (VA-LRTI), and their impact on outcome.

Methods: This retrospective study was performed in five 10-bed ICUs of a teaching hospital, during a 2-year period. Ventilator-associated lower respiratory tract infections (VA-LRTI), including ventilator-associated tracheobronchitis (VAT) and ventilator-associated pneumonia (VAP) were prospectively diagnosed. The agreement between VAE, VAT and VAP was assessed by k statistics.

Results: A total of 1059 patients (15,029 ventilator-days) were included. 268 VAP (17.8 per 1000 ventilator-days), 127 VAT (8.5 per 1000 ventilator-days) and 262 VAE (17.4 per 1000 ventilator-days) were diagnosed. There was no agreement between VAT and VAE, and the agreement was poor between VAP and VAE $(k=0.12,95 \% \mathrm{Cl} 0.03-0.20)$. VAE and VA-LRTI were associated with significantly longer duration of mechanical ventilation, ICU and hospital length of stay. VAP, VAT and VAE were not significantly associated with mortality in multivariate analysis.

Conclusions: The agreement was poor between VAE and VAP. No agreement was found between VAE and VAT. VAE episodes were significantly associated with longer duration of mechanical ventilation and length of stay, but not with ICU mortality.
\end{abstract}

Keywords: Ventilator-associated events, Ventilator-associated tracheobronchitis, Ventilator-associated pneumonia

\section{Background}

In spite of increased use of non-invasive mechanical ventilation, and high-flow nasal oxygen in the intensive care unit (ICU), invasive mechanical ventilation is still used in a large proportion of critically ill patients [1]. Ventilator-associated lower respiratory tract infections (VALRTI), including ventilator-associated pneumonia (VAP), and ventilator-associated tracheobronchitis (VAT) are the most common complications in patients receiving mechanical ventilation. These infections are associated

\footnotetext{
*Correspondence: s-nseir@chru-lille.fr

${ }^{1}$ Critical Care Center, CHU Lille, 59000 Lille, France

Full list of author information is available at the end of the article
}

with increased duration of mechanical ventilation, length of hospital stay, and cost $[2,3]$.

The diagnosis of these infections is based on chest $\mathrm{X}$-ray, which is not specific in detecting new infiltrates in critically ill patients. Therefore, the CDC recommended using a new definition for ventilator-associated events (VAE), including infectious and other conditions. This definition includes only objective criteria and is perfectly reproducible [4]. However, recent studies and meta-analysis reported poor agreement between VAE, including ventilator-associated conditions (VAC), infection-related ventilator-associated complications (IVAC), or probable VAP (pVAP) [5-7]. Few studies evaluated the agreement between VAE and VA-LRTI, including VAP and VAT. Although VAP and VAT are both associated with 
increased duration of mechanical ventilation and length of ICU stay, only VAP is associated with increased mortality rates [8]. Thus, it is probably important to distinguish VAP from VAT. In addition, the recent ATS/IDSA guidelines on VAP recommended not treating VAT patients with antimicrobial, based on the low quality of the available evidence [9]. Therefore, we conducted this retrospective analysis of prospectively collected data to determine the agreement between VAE and VA-LRTI, including VAP and VAT. We also aimed to determine the impact of VAE on outcomes, including duration of mechanical ventilation, length of ICU and hospital stay, and mortality.

\section{Patients and methods \\ Study design}

This study was conducted in five 10-bed ICUs in Lille University Hospital, during a 2-year period (from January 1st, 2016 through December 31st, 2017). The IRB of the Lille University Hospital approved the study and waived informed consent. In accordance with the French law, and because of the retrospective observational design, written informed consent was not required. All patients hospitalized in one of the 5 ICUs and receiving invasive mechanical ventilation for at least 5 days were eligible for this study. Patients who received mechanical ventilation for $<5$ days, and those who received mechanical ventilation for $>24 \mathrm{~h}$ before ICU admission were excluded.

\section{Definitions}

VA-LRTI included VAP and VAT. VAP was defined as pneumonia diagnosed after $48 \mathrm{~h}$ of intubation and mechanical ventilation. The diagnostic criteria for VAP included a new infiltrate on chest X-ray associated with at least two of the following: body temperature $\geq 38.5^{\circ} \mathrm{C}$ or $<36{ }^{\circ} \mathrm{C}$; leukocyte count $\geq 10 \times 10^{9} / \mathrm{L}$ or $<1.5 \times 10^{9} / \mathrm{L}$; and purulent tracheal aspirate or sputum. In addition, a microbiological confirmation was required for all patients (positive endotracheal aspirate culture $\geq 10^{5}$ colony-forming units $(\mathrm{cfu}) / \mathrm{mL}$ or positive bronchoalveolar lavage culture $\geq 10^{4} \mathrm{cfu} / \mathrm{mL}$ ) [9]. VAT was defined using the same criteria as for VAP, except the presence of new or progressive pulmonary infiltrate. VAE were diagnosed according to CDC definition (Additional file 1: Figure S1). VAE diagnosis was considered concordant with that of VAT or VAP, when these infections occurred within 2 days before or after the alteration of PEEP or FiO2 (Additional file 1: Figure S2).

\section{VAP prevention and treatment}

A VAP prevention strategy was routinely used during the study period. The ventilator circuit was not changed routinely. Sedation and weaning were based on a written protocol. A minimal positive end expiratory pressure of $5 \mathrm{~cm} \mathrm{H}_{2} \mathrm{O}$ was used in all patients. Oral cavity was cleaned with chlorhexidine thrice daily. Cuff pressure was measured and adjusted $\left(25 \mathrm{~cm} \mathrm{H}_{2} \mathrm{O}\right)$ by nurses thrice a day. Tracheal suctioning was routinely performed by nurses, using an open tracheal suction system. Patients remained in semi-recumbent position, and received enteral nutrition based on a written protocol.

Antibiotic treatment for patients with suspected VAP was based on ATS/IDSA guidelines [9]. Antibiotic treatment for other infections was based on written local guidelines adapted from international and national guidelines. Stress ulcer prophylaxis was not routinely used. Selective digestive decontamination was not used.

\section{Data collection}

Data related to VAP and VAT episodes were prospectively collected. Data regarding mechanical ventilation (PEEP and FiO2) were automatically imported every hour in the patient management software ICIP ${ }^{\circledR}$ (Philips Healthcare). Data from 2-h time slots were then retrospectively examined to determine the episodes of VAE. Other data such as body temperature, leukocytosis, antibiotic use, patient characteristics, aetiology of VAE episodes, duration of mechanical ventilation and hospitalization, and mortality were collected retrospectively from patients' computerized medical records.

\section{Statistical analysis}

The incidence rate and Cohen's kappa coefficients were calculated on all episodes of VAE, VAP and VAT. The concordance between the diagnosis for VAC, IVAC, pVAP and that of VAP and VAT was determined by Cohen's kappa statistic [10].

Only first episodes were taken to examine patients' characteristics according to the occurrence of VAE, VAP and VAT. Qualitative variables were expressed in percentage. Because of non-normal distribution, quantitative variables were expressed in median, 25th and 75th percentiles. Chi squared test, or Fisher's exact test; and Mann-Whitney U test, or Kruskal-Wallis test, were used to compare the qualitative and quantitative variables, respectively. The difference was considered significant when $p<0.05$. When a significant difference existed between patients with VAP, VAT, and those with no VALRTI, comparisons between different groups were performed: VAP vs VAT, VAP vs no VA-LRTI and VAT vs no VA-LRTI.

Multivariate analyses, using forward multiple logistic regression models, were performed to determine the impact of VA-LRTI and VAE on mortality, adjusting for age, SAPS II and immunosuppression. 


\section{Results}

Out of the 1896 patients who received invasive mechanical ventilation, 837 (44\%) were excluded (Fig. 1). Overall 1059 patients were included and received 15,029 days of invasive mechanical ventilation.

\section{Incidence of VAE and VA-LRTI}

A total of 262 episodes of VAC (17.4 for 1000 ventilatordays), 268 VAP (17.8 for 1000 ventilator-days) and 127 VAT ( 8.5 for 1000 ventilator-days) were diagnosed and used for concordance analysis.

At least one episode of VAC, IVAC, and pVAP was diagnosed in 227 (21.4\%), 123 (11.6\%), and 62 (5.9\%) patients, respectively. At least one episode of VAT or VAP were diagnosed in 99 (9.3\%) and $215(20.3 \%)$ patients, respectively. Among patients with VAT, 12 (9.4\%) patients developed a subsequent VAP.

\section{Correlation between VAE and VA-LRTI}

A total of 6 VAT and 71 VAP were correlated with VAC (Fig. 2). Concordance (kappa statistic) between VAC and VAP, IVAC and VAP, pVAP and VAP were 0.03 (95\% CI 0-0.11), 0.15 (95\% CI 0.07-0.23), and 0.27 (95 CI 0.180.35 ), respectively (Table 1 ). Because of the small number of patients with VAE and VAT $(n=6)$, kappa statistic could not be calculated.

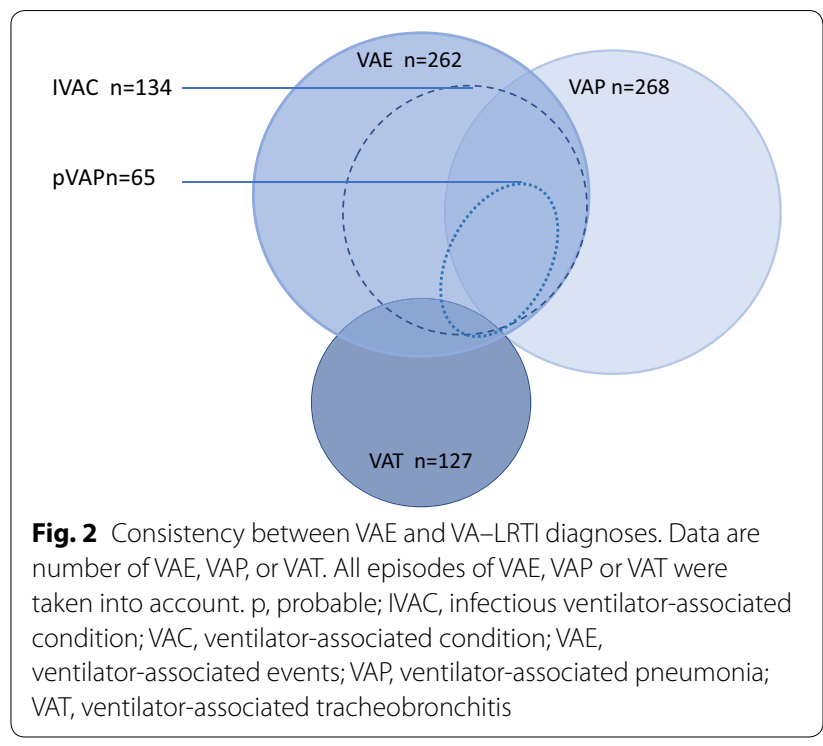

\section{Clinical significance of VAE}

The most common causes for VAE were VAP $(n=79$, $30 \%)$, and atelectasis $(n=53,20 \%) .2 .6 \%$ of VAE were possibly related to VAT, and no aetiology was found for (63, 24\%) episodes (Fig. 3).

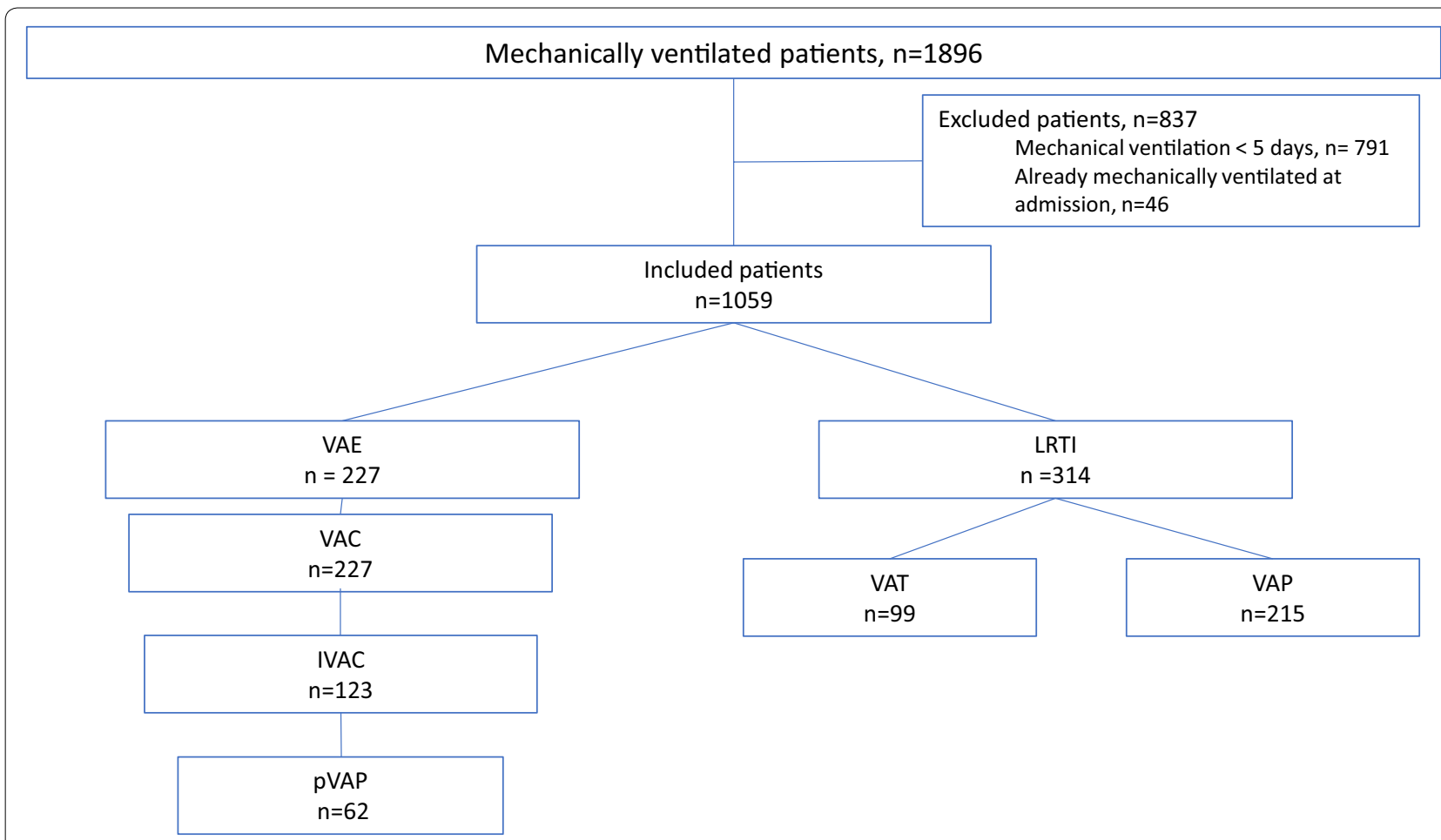

Fig. 1 Flowchart. Data are number of patients. p, probable; IVAC, infectious ventilator-associated condition; VAC, ventilator-associated condition; VAP, ventilator-associated pneumonia; VAT, ventilator-associated tracheobronchitis 
Table 1 Correlation between ventilator-associated events and ventilator-associated lower respiratory tract infections

\begin{tabular}{|c|c|c|c|}
\hline & VAC, $n=262$ & IVAC, $n=134$ & pVAP, $n=65$ \\
\hline VAP & 71 (27.1) & $58(43.3)$ & $56(86.2)$ \\
\hline \multirow[t]{2}{*}{ No VAP } & 191 (72.9) & 76 (56.7) & $9(13.8)$ \\
\hline & $\mathrm{K}_{\mathrm{VAP}-\mathrm{VAC}}=0.03(0-0.11)$ & $\mathrm{K}_{\mathrm{VAP}-\mathrm{IVAC}}=0.15(0.07-0.23)$ & $\mathrm{K}_{\mathrm{VAP}-\mathrm{pVAP}}=0.27(0.18-0.35)$ \\
\hline VAT & $6(2.3)$ & $3(2.2)$ & $3(4.6)$ \\
\hline No VAT & $256(97.7)$ & $131(97.8)$ & $62(95.4)$ \\
\hline
\end{tabular}

Data are numbers (\%)

The correlation between ventilator-associated events and VAT could not be calculated because of the small number of patients with VAC and VAT ( $n=6$ )

IVAC, infection-related ventilator-associated condition; $\mathrm{p}$, probable; VAC, ventilator-associated condition; VAP, ventilator-associated pneumonia; VAT, ventilatorassociated tracheobronchitis

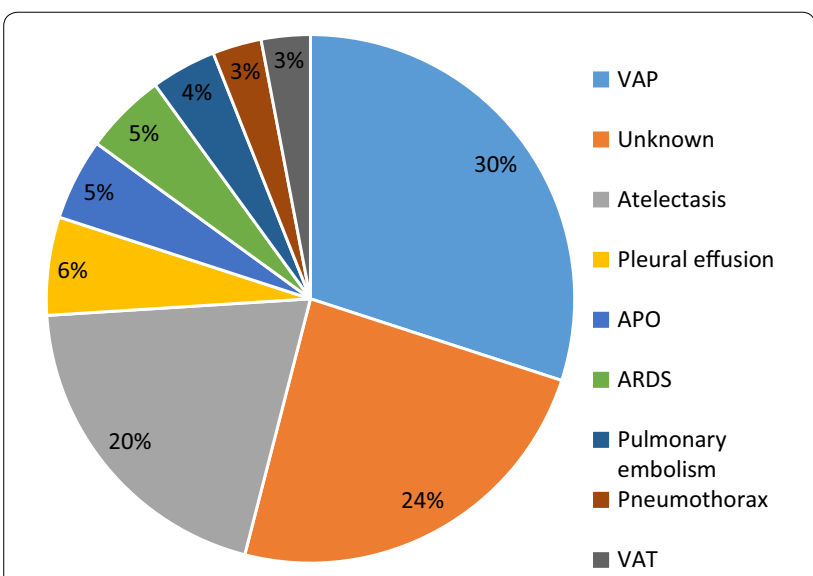

Fig. 3 Clinical causes for VAE. APO, acute pulmonary oedema; ARDS, acute respiratory distress syndrome; VAP, ventilator-associated pneumonia; VAT, ventilator-associated tracheobronchitis

\section{Patient characteristics based on VA-LRTI}

Characteristics of patients who presented VAT, VAP, or no VA-LRTI are presented in Table 2. A significant difference was found between the three groups regarding male gender, age, neurologic failure at ICU admission, and immunosuppression. Median duration of mechanical ventilation before VAT, and VAP occurrence was 9 (5, $13)$, and $7(4,13)$ days, respectively.

\section{Patient characteristics based on VAE}

The characteristics of patients with VAC, IVAC, or pVAP, and those without these conditions are presented in Table 3. In VAC patients, as compared with those with no VAC, male gender, SOFA at ICU admission, and BMI > 30 were significantly higher. In IVAC patients, as compared with those with no IVAC, SOFA score at ICU admission, and $\mathrm{BMI}>30$ were significantly higher. In pVAP patients, as compared with those with no pVAP, age, medical category, and Charlson comorbidity index were significantly lower. Median duration of mechanical ventilation before
VAC, and iVAC, and pVAP occurrence was $5(3,9), 5$ (3, $9)$, and $5(4,10)$ days, respectively.

\section{Outcomes based on VA-LRTI}

ICU mortality, duration of mechanical ventilation and length of stay were significantly different between patients with VAP, VAT, or no VA-LRTI (Table 4). In patients with VAP, as compared with those with no VALRTI, duration of mechanical ventilation, and length of stay were significantly higher. In patients with VAP, as compared with those with VAT, ICU mortality was significantly higher. In patients with VAT, as compared with those with no VA-LRTI, duration of mechanical ventilation and length of stay were higher, and ICU mortality was significantly lower. In multivariate analysis, the occurrence of VA-LRTI was not associated with mortality (Table 6).

\section{Outcomes based on VAE}

Although duration of mechanical ventilation and length of stay were significantly higher in patients with VAC, IVAC, or pVAC, as compared with those without these conditions, no significant difference in mortality was found between these different groups (Table 5). In multivariate analysis, the occurrence of VAE, or of VA-LRTI was not associated with mortality (Table 6).

\section{Discussion}

Our results suggest that VAE are moderately correlated to VAP, and not correlated to VAT. VAE and VA-LRTI are all associated with increased duration of mechanical ventilation and length of hospital and ICU stay. VA-LRTI and VAE were not independently associated with mortality.

The strengths of our study include the large number of included patients $(n=1059)$ and ventilator days $(\mathrm{n}=15,029)$, and the prospective evaluation of all VALRTI, including VAT. Previous studies reported similar findings regarding the correlation between VAE and 
Table 2 Patient characteristics at ICU admission, based on the presence of ventilator-associated lower respiratory tract infections

\begin{tabular}{|c|c|c|c|c|}
\hline & VAP, $n=215$ & VAT, $n=99$ & No VA-LRTI, $n=745$ & $p$ \\
\hline Male gender & $156(72.5)$ & $66(66.6)$ & $473(63.5)$ & $0.001^{\#}$ \\
\hline Age, years & $57(45-67)$ & $59(47-69)$ & $61(49-69.8)$ & $0.041^{\#}$ \\
\hline SAPS II & $59(48-73)$ & $55(45-68)$ & $58.5(46-71)$ & 0.126 \\
\hline SOFA & $10(7-12)$ & $9(6-11)$ & $9(6-12)$ & 0.091 \\
\hline \multicolumn{5}{|l|}{ Reason for admission } \\
\hline Medical vs surgical & $181(84.2)$ & $80(80.8)$ & $622(83.5)$ & 0.742 \\
\hline Septic shock & $54(25.1)$ & $17(17.2)$ & $194(26)$ & 0.158 \\
\hline Acute respiratory failure & $80(37.2)$ & $35(35.4)$ & $260(34,9)$ & 0.830 \\
\hline Neurologic failure & $53(24.7)$ & $28(28.3)$ & $129(17.3)$ & $0.005^{\# *}$ \\
\hline Soft tissue infection & $15(7)$ & $12(12.1)$ & 79 (10.6) & 0.224 \\
\hline Cardiac arrest & $22(10.2)$ & $10(10.1)$ & $88(11.8)$ & 0.741 \\
\hline Poisoning & $8(3.7)$ & $2(2)$ & $37(5)$ & 0.345 \\
\hline Other & $35(16.3)$ & $12(12.1)$ & $115(15.4)$ & 0.624 \\
\hline Charlson comorbidity index & $3(1-5)$ & $3(1-5)$ & $4(2-6)$ & 0.660 \\
\hline Diabetes & $43(20)$ & $20(20.2)$ & $177(23.8)$ & 0.417 \\
\hline Chronic renal failure & $17(7.9)$ & $13(13.1)$ & $75(10.1)$ & 0.343 \\
\hline Chronic heart failure & $37(17.2)$ & $21(21.2)$ & $132(17.7)$ & 0.665 \\
\hline Chronic respiratory failure & $15(15.9)$ & $7(10.9)$ & $57(14.7)$ & 0.885 \\
\hline Cirrhosis & $18(8.4)$ & $8(8)$ & $67(7.7)$ & 0.918 \\
\hline Immunosuppression & $59(27.4)$ & $17(17.2)$ & $215(28.9)$ & $0.049^{* !}$ \\
\hline \multicolumn{5}{|l|}{ Obesity } \\
\hline $\mathrm{BMI}>30$ & $50(23.3)$ & $26(26.3)$ & $141(18.9)$ & 0.128 \\
\hline $\mathrm{BMI}>35$ & $27(12.6)$ & $11(11.1)$ & $78(10.5)$ & 0.320 \\
\hline
\end{tabular}

Results are numbers (\%), or median (interquartile range)

BMI: body mass index; SAPS, simplified acute physiology score; SOFA, sepsis-related organ failure assessment; VA-LRTI, ventilator-associated low respiratory tract infection; VAP, ventilator-associated pneumonia; VAT, ventilator-associated tracheobronchitis

* Significant difference between VAT and no VA-LRTI groups

\# Significant difference between VAP and no VA-LRTI groups

a Significant difference between VAP and VAT groups

VAP $[6,11,12]$. However, few studies prospectively evaluated the incidence of VAT in patients with VAE [11, 13]. Among the 262 diagnosed episodes of VAE, only 6 (2\%) were possibly related to VAT. Thus, the correlation between VAE, and VAT could not be calculated. In a retrospective analysis of prospectively collected data, Bouadma et al. [11] identified aetiologies of each episode and found only $1 \%$ of VAC caused by tracheobronchitis.

Worsening of ventilatory parameters is not a mandatory criterion for VA-LRTI definition, and only the Clinical Pulmonary Infection Score (CPIS) includes the alteration of the $\mathrm{PaO}_{2} / \mathrm{FiO}_{2}$. Our study and previous studies $[5,11]$ reported that an important percentage of VAE episodes were possibly related to VAP, and not to VAT. This clearly suggests that using alteration of oxygenation, i.e. $\mathrm{PaO}_{2} / \mathrm{FiO}_{2}$, could be helpful in differentiating VAP from VAT. Differentiating these two infections could be a difficult task, as the accuracy of chest X-ray in diagnosing new infiltrates is low [14-16].
However, it is still important to differentiate them, as antibiotic treatment is not recommended for VAT and inappropriate use of antimicrobials is a risk factor for subsequent emergence of multidrug-resistant bacteria $[17,18]$. Previous large observational studies and two small randomized controlled trials suggested beneficial effects of systemic and inhaled antibiotics. However, several limitations preclude definite conclusions on the interest of antimicrobials in patients with VAT, and further large multicentre randomized controlled trials are required.

The incidence of VAE in our study is in line with previous findings. However, the incidence of VAP (21\%) is somehow higher than that reported by recent studies [19]. This could be explained by the fact that only patients receiving mechanical ventilation for $>4$ days were included in our study.

VAE, and VA-LRTI were associated with significantly longer duration of mechanical ventilation and hospital 
Table 3 Patient characteristics at ICU admission, based on the presence of ventilator-associated events

\begin{tabular}{|c|c|c|c|c|c|c|c|c|c|}
\hline & VAC, $n=227$ & No VAC, $n=832$ & $p$ & IVAC, $n=123$ & No IVAC, $n=936$ & $p$ & pVAP, $n=62$ & No $p V A P, n=997$ & $p$ \\
\hline Male gender & $164(72.2)$ & $531(63.8)$ & 0.020 & $88(71.5)$ & $607(64,9)$ & 0.150 & $44(71)$ & $651(65.3)$ & 0.372 \\
\hline Age, years & $59(47-67)$ & $61(47-70)$ & 0.227 & $58(46-67)$ & $61(47-69.7)$ & 0.121 & $55(45-66)$ & $61(47-70)$ & 0.006 \\
\hline SAPS $\|$ & $56(46-74)$ & $59(46-70)$ & 0.804 & $58(44-72)$ & $58(46-70)$ & 0.353 & $60(47-73)$ & $58(46-71)$ & 0.192 \\
\hline SOFA & $9(7-12)$ & $9(6-11)$ & 0.041 & $9(6-12)$ & $10(6.25-11)$ & 0.009 & $9(7-13)$ & $9(7-12)$ & 0.392 \\
\hline \multicolumn{10}{|l|}{ Reason for admission } \\
\hline Medical vs surgical & $189(83.3)$ & $693(83.3)$ & 0.933 & $94(76.4)$ & $788(84.2)$ & 0.026 & $46(74.2)$ & $836(83,9)$ & 0.043 \\
\hline Septic shock & 65 (28.6) & $200(24)$ & 0.162 & $32(26)$ & $233(24.9)$ & 0.797 & $14(22.6)$ & $251(25.1)$ & 0.641 \\
\hline $\begin{array}{l}\text { Acute respiratory } \\
\text { failure }\end{array}$ & $93(41)$ & $282(33,9)$ & 0.051 & $43(35)$ & $332(35.5)$ & 0.898 & $18(29)$ & $357(35.8)$ & 0.274 \\
\hline Neurologic failure & $39(17.2)$ & $171(20.6)$ & 0.252 & $21(17.1)$ & $189(20.2)$ & 0.409 & $11(17.7)$ & $199(20)$ & 0.665 \\
\hline Soft tissue infection & $23(10.1)$ & $83(10)$ & 0.953 & $16(13)$ & $90(9.6)$ & 0.242 & $8(12.9)$ & $98(9.8)$ & 0.437 \\
\hline Cardiac arrest & $21(9.3)$ & $98(11.8)$ & 0278 & $13(10.1)$ & $106(11.3)$ & 0.794 & $9(14.5)$ & $110(11.1)$ & 0.405 \\
\hline Poisoning & $7(3.1)$ & $40(4.8)$ & 0.261 & $4(3.3)$ & $43(4.6)$ & 0.343 & $2(3.2)$ & $45(4.5)$ & 0.631 \\
\hline Other & $44(19.4)$ & $118(14.2)$ & 0.056 & $28(22.8)$ & $134(14.3)$ & 0.015 & $12(19.4)$ & $150(15.1)$ & 0.364 \\
\hline $\begin{array}{l}\text { Charlson comorbidity } \\
\text { index }\end{array}$ & $3(2-5)$ & $4(1-5)$ & 0.440 & $3(1-5)$ & $4(2-5)$ & 0.639 & $3(2-5)$ & $4(1-5)$ & 0.024 \\
\hline Diabetes & $58(25.6)$ & $182(21.9)$ & 0.248 & $33(26.8)$ & $207(22.2)$ & 0.246 & $13(21)$ & $227(22.8)$ & 0.736 \\
\hline Chronic renal failure & $17(7.5)$ & 88 (10.6) & 0.899 & $9(7.3)$ & $96(10.3)$ & 0.302 & $3(4.8)$ & $102(10.3)$ & 0.167 \\
\hline Chronic heart failure & $40(17.6)$ & $150(18.1)$ & 0.875 & $22(17.9)$ & $168(18)$ & 0.978 & $10(16.1)$ & $180(18.1)$ & 0.696 \\
\hline $\begin{array}{l}\text { Chronic respiratory } \\
\text { failure }\end{array}$ & $36(15.9)$ & $120(14.5)$ & 0.598 & $19(15.4)$ & $137(14.7)$ & 0.819 & $10(16.1)$ & $146(14.7)$ & 0.754 \\
\hline Cirrhosis & $23(10.1)$ & $70(8.4)$ & 0.436 & $13(10.6)$ & $80(8.6)$ & 0.470 & $6(9.7)$ & $87(8.7)$ & 0.809 \\
\hline Immunosuppression & $55(24.2)$ & $236(28.4)$ & 0.209 & $33(26.8)$ & $258(27.6)$ & 0.853 & $17(27.4)$ & $274(27.5)$ & 0.984 \\
\hline \multicolumn{10}{|l|}{ Obesity } \\
\hline $\mathrm{BMI}>30$ & $64(28.2)$ & $153(18.4)$ & 0.001 & $35(28.5)$ & $182(19.5)$ & 0.021 & $8(29)$ & $199(20)$ & 0.088 \\
\hline $\mathrm{BMI}>35$ & $30(13.2)$ & $86(10.3)$ & 0.223 & 18 (14.6) & $98(10.5)$ & 0.167 & $10(16.1)$ & 106 (10.6) & 0.181 \\
\hline
\end{tabular}

Data are numbers (\%), or median (interquartile range)

BMI, body mass index; SAPS: simplified acute physiology score; SOFA, sepsis-related organ failure assessment

Table 4 Outcomes of study patients based on the presence of ventilator-associated lower respiratory tract infections

\begin{tabular}{lcccc}
\hline & VAP, $\boldsymbol{n = 2 1 5}$ & VAT, $\boldsymbol{n = 9 9}$ & No VA-LRTI, $\boldsymbol{n = 7 4 5}$ & $\boldsymbol{p}$ \\
\hline ICU mortality & $82(38.1)$ & $20(20.2)$ & $313(42)$ & $<0.001^{* \text { * }}$ \\
Duration of mechanical ventilation, days & $18(11-31)$ & $18(11-24)$ & $8(0-13)$ & $<0.001^{*}$ \\
Mechanical ventilation-free days & $1(0-13)$ & $25(17-37)$ & $11(0-20)$ & $<0.001^{\# * a}$ \\
Length of ICU stay, days & $26(15-42)$ & $36(24-56.3)$ & $12(8-18)$ & $<0.001^{\# *}$ \\
Length of hospital stay, days & $31(18-60)$ & $10-30)$ & $<0.001^{\# *}$ \\
\hline
\end{tabular}

Data are numbers (\%), or median (interquartile range)

d, days; VAP, ventilator-associated pneumonia; VAT, ventilator-associated tracheobronchitis

* Significant difference between VAT and no VA-LRTI groups

\# Significant difference between VAP and no VA-LRTI groups

" Significant difference between VAP and VAT groups

and ICU stay. However, VAE and VA-LRTI were not independently associated with mortality. In contrast, two previous studies reported that VAE were associated with significantly higher mortality rates $[11,12]$.

VAE diagnosis algorithm is based on objective criteria and easy to use in routine in mechanically ventilated patients. However, the clinical relevance of VAE is not clear. First, our study and previous ones clearly showed that applying a VAE algorithm surveillance is not accurate in detecting VA-LRTI. Second, the impact of a ventilator bundle on VAE incidence is unknown. Few studies have focused on the preventability of VAE, but it seems 
Table 5 Outcomes of study patients based on the presence of ventilator-associated events

\begin{tabular}{|c|c|c|c|c|c|c|c|c|c|}
\hline & VAC, $n=227$ & No VAC, $n=832$ & $p$ & IVAC, $n=123$ & No IVAC, $n=936$ & $p$ & pVAP, $n=62$ & No pVAP, $n=995$ & $p$ \\
\hline ICU mortality & $98(43.2)$ & $316(38)$ & 0.163 & $23(45.1)$ & $340(36.3)$ & 0.180 & $26(41.9)$ & 388 (39) & 0.547 \\
\hline $\begin{array}{l}\text { Duration of } \\
\text { mechanical venti- } \\
\text { lation, days }\end{array}$ & $15(10-27)$ & $9(6-15)$ & $<0.001$ & $16(12.5-29)$ & $9(6-16)$ & $<0.001$ & $18(15-31)$ & $9(6-16)$ & $<0.001$ \\
\hline $\begin{array}{l}\text { Mechanical } \\
\text { ventilation-free } \\
\text { days }\end{array}$ & $1(0-11)$ & $9(0-16)$ & $<0.001$ & $1(0-10)$ & $8(0-15)$ & $<0.001$ & $5(0-14)$ & $8(0-15)$ & 0.002 \\
\hline $\begin{array}{l}\text { Length of ICU stay, } \\
\text { days }\end{array}$ & $19(13-32)$ & $14(9-23)$ & $<0.001$ & $20(16-31.5)$ & $14(9-24)$ & $<0.001$ & $23(19-34)$ & $14(9-24)$ & $<0.001$ \\
\hline $\begin{array}{l}\text { Length of hospital } \\
\text { stay, days }\end{array}$ & $27(17-51)$ & $19(11-35)$ & $<0.001$ & $27(18.5-52)$ & $20(11-35.5)$ & $<0.001$ & $28(19.5-53)$ & $20(11-36)$ & $<0.001$ \\
\hline
\end{tabular}

Data are numbers (\%), or median (interquartile range)

$\mathrm{d}$, day; IVAC, infection-related ventilator-associated condition; p, probable; VAC, ventilator-associated condition

Table 6 Risk factor for ICU mortality by multivariate analysis

\begin{tabular}{lll}
\hline & OR $(\mathbf{9 5} \% \mathrm{Cl})$ & $\boldsymbol{p}$ value \\
\hline Model 1 & & \\
Immunosuppression & $2.1(1.6-2.8)$ & $<0.001$ \\
Age, years & $1.02(1.01-1.03)$ & $<0.001$ \\
SAPS II & $1.02(1.01-1.04)$ & $<0.001$ \\
VA-LRTI & & 0.128 \\
VAT & - & - \\
VAP & - & - \\
$\quad$ No infection & - & - \\
Model 2 & & $<0.001$ \\
Immunosuppression & $2.1(1.6-2.8)$ & $<0.001$ \\
Age, years & $1.02(1.01-1.03)$ & $<0.001$ \\
SAPS II & $1.02(1.01-1.04)$ & 0.149 \\
VAE & - & \\
\hline
\end{tabular}

Hosmer and Lemeshow goodness-of-fit test $p=0.39$, and $p=0.46$ for models 1 , and 2, respectively

SAPS, simplified acute physiology score; VA-LRTI, ventilator-associated lower respiratory tract infections; VAE, ventilator-associated events

that bundles applied for VAP prevention are not completely effective for VAE prevention [20-22]. Our results show that many VAE were non-infectious events as atelectasis, pleural effusion or acute pulmonary embolism. This might explain why ventilator bundles are not effective in preventing VAE, and should not be used to assess the quality of care in mechanically ventilated patients.

VAE algorithm failed in identifying most of VAT and 73\% of VAP episodes and should not be used to start an empirical treatment of VA-LRTI. Furthermore, the retrospective nature of VAE does not allow its use at bedside. In fact, to meet IVAC criteria, patients must have 2 days of stable oxygenation parameter and 2 days of worsening ventilatory settings after which a new antibiotic must be prescribed for at least 4 days.
Our study has some limitations. First, it was performed in a single center, and its results may not be generalized to all ICU patients. Second, it was retrospective. However, all VA-LRTI were prospectively identified. Third, no data were collected on duration of antibiotic treatment before VA-LRTI, and VAE, neither on appropriateness of antibiotic treatment in patients with VA-LRTI. Fourth, the definition of VAT and VAP was based on chest X-ray that was interpreted by physicians in charge and no blind interpretation was performed. The prolonged duration of mechanical ventilation reported in patients with VA-LRTI, or VAE, as compared to those with no infection, or no VAE could not be attributed to these events as no adjustment was performed.

\section{Conclusion}

VAE and VA-LRTI are common in mechanically ventilated critically ill patients, and have a significant impact on duration of mechanical ventilation and length of stay. VAE are moderately correlated to VAP, and not correlated to VAT. Our results suggest that VAE should not be used as a marker of quality of care or to start empirical antibiotic treatment.

\section{Supplementary information}

Supplementary information accompanies this paper at https://doi. org/10.1186/s13613-020-0624-6.

Additional file 1. Online supplementary material.

\section{Abbreviations}

$\mathrm{Cl}$ : confidence interval; CPIS: clinical pulmonary infection score; OR: odds ratio; RGV: residual gastric volume; VAP: ventilator-associated pneumonia; VAT: ventilator-associated tracheobronchitis.

\section{Acknowledgements}

None. 


\section{Authors' contribution}

SN, and AR designed the study. OP, and SN performed statistical analyses. All authors collected the data, contributed in interpretation of the results, drafted and approved the submitted manuscript. SN had full access to the data and takes the responsibility for the integrity of the data and the accuracy of the data analysis. OP and SL contributed equally to this study. All authors read and approved the final manuscript.

\section{Funding}

None.

\section{Availability of data and materials}

All data are provided in the manuscript.

\section{Ethics approval and consent to participate}

The IRB of the Lille University Hospital approved the study and waived informed consent. In accordance with the French law, and because of the retrospective observational design, written informed consent was not required.

\section{Consent for publication}

Not applicable.

\section{Competing interests}

SN: MSD (advisory board and lecture); Pfizer, Gilead, Bio Rad, and Biomérieux (lecture). AR: Maatpharma (advisory board); Pfizer (lecture). Other authors: none.

\section{Author details}

${ }^{1}$ Critical Care Center, CHU Lille, 59000 Lille, France. ${ }^{2}$ Medicine Faculty, Lille University, 59000 Lille, France. ${ }^{3}$ Intensive Care Unit, $\mathrm{CH}$ de Boulogne, Boulogne, France. ${ }^{4}$ Centre de Biologie et de Pathologie, CHU Lille, 59000 Lille, France.

Received: 23 April 2019 Accepted: 7 January 2020

Published online: 13 January 2020

\section{References}

1. Nosocomial infection surveillance in intensive care units. REA-Raisin network, France-Results 2017. https://www.santepubliquefrance.fr/docs/ surveillance-des-infections-nosocomiales-en-reanimation-adulte-resea u-rea-raisin-france-resultats-2017. Accessed 22 Apr 2019.

2. Safdar N, Dezfulian C, Collard H, Saint S. Clinical and economic consequences of ventilator-associated pneumonia: a systematic review. Crit Care Med. 2005:33(10):2184-93.

3. Martin-Loeches I, Povoa P, Rodríguez A, Curcio D, Suarez D, Mira J-P, et al. Incidence and prognosis of ventilator-associated tracheobronchitis (TAVeM): a multicentre, prospective, observational study. Lancet Respir Med. 2015;3(11):859-68.

4. Nuckchady D, Heckman MG, Diehl NN, Creech T, Carey D, Domnick $R$, et al. Assessment of an automated surveillance system for detection of initial ventilator-associated events. Am J Infect Control. 2015;43(10):1119-21.

5. Fan Y, Gao F, Wu Y, Zhang J, Zhu M, Xiong L. Does ventilator-associated event surveillance detect ventilator-associated pneumonia in intensive care units? A systematic review and meta-analysis. Crit Care. 2016;20(1):338

6. Klein Klouwenberg PMC, van Mourik MSM, Ong DSY, Horn J, Schultz MJ, Cremer OL, et al. Electronic implementation of a novel surveillance paradigm for ventilator-associated events. Feasibility and validation. Am J Respir Crit Care Med. 2014;189(8):947-55.

7. Nair GB, Niederman MS. Ventilator-associated pneumonia: present understanding and ongoing debates. Intensive Care Med. 2015;41(1):34-48.

8. Melsen WG, Rovers MM, Groenwold RH, Bergmans DC, Camus C, Bauer $\mathrm{TT}$, et al. Attributable mortality of ventilator-associated pneumonia: a meta-analysis of individual patient data from randomised prevention studies. Lancet Infect Dis. 2013;13(8):665-71.

9. Kalil AC, Metersky ML, Klompas M, Muscedere J, Sweeney DA, Palmer LB, et al. Management of Adults with hospital-acquired and ventilatorassociated pneumonia: 2016 Clinical Practice Guidelines by the Infectious Diseases Society of America and the American Thoracic Society. Clin Infect Dis. 2016;63(5):e61-111.

10. McHugh ML. Interrater reliability: the kappa statistic. Biochem Med. 2012;22(3):276-82.

11. Bouadma L, Sonneville R, Garrouste-Orgeas M, Darmon M, Souweine B, Voiriot G, et al. Ventilator-associated events: prevalence, outcome, and relationship with ventilator-associated pneumonia. Crit Care Med. 2015;43(9):1798-806.

12. Muscedere J, Sinuff T, Heyland DK, Dodek PM, Keenan SP, Wood G, et al. The clinical impact and preventability of ventilator-associated conditions in critically ill patients who are mechanically ventilated. Chest. 2013;144(5):1453-60

13. the EU-VAE Study Investigators Group, Ramírez-Estrada S, Lagunes L, Peña-López Y, Vahedian-Azimi A, Nseir S, et al. Assessing predictive accuracy for outcomes of ventilator-associated events in an international cohort: the EUVAE study. Intensive Care Med. 2018:44(8):1212-20.

14. Butler KL. The chest radiograph in critically ill surgical patients is inaccurate in predicting ventilator-associated pneumonia. Am Surg. 1999;40:805-9

15. Hayden GE, Wrenn KW. Chest radiograph vs. computed tomography scan in the evaluation for pneumonia. J Emerg Med. 2009;36(3):266-70.

16. Self WH, Courtney DM, McNaughton CD, Wunderink RG, Kline JA. High discordance of chest X-ray and computed tomography for detection of pulmonary opacities in ED patients: implications for diagnosing pneumonia. Am J Emerg Med. 2013;31(2):401-5.

17. Vincent J-L. International study of the prevalence and outcomes of infection in intensive care units. JAMA. 2009;302(21):2323.

18. Barbier F, Lisboa T, Nseir S. Understanding why resistant bacteria are associated with higher mortality in ICU patients. Intensive Care Med. 2016:42(12):2066-9

19. Koulenti D, Tsigou E, Rello J. Nosocomial pneumonia in 27 ICUs in Europe: perspectives from the EU-VAP/CAP study. Eur J Clin Microbiol Infect Dis. 2017;36(11):1999-2006.

20. Kallet $\mathrm{RH}$. Ventilator bundles in transition: from prevention of ventilatorassociated pneumonia to prevention of ventilator-associated events. Respir Care. 2019;64(8):994-1006.

21. Klompas M, Anderson D, Trick W, Babcock H, Kerlin MP, Li L, et al. The preventability of ventilator-associated events. The CDC Prevention Epicenters Wake Up and Breathe Collaborative. Am J Respir Crit Care Med. 2015;191(3):292-301.

22. Cocoros NM, Klompas M. Ventilator-associated events and their prevention. Infect Dis Clin North Am. 2016;30(4):887-908.

\section{Publisher's Note}

Springer Nature remains neutral with regard to jurisdictional claims in published maps and institutional affiliations. 